\title{
Energy-consistent, Galerkin approach for the nonlinear dynamics of beams using intrinsic equations
}

\author{
Mayuresh J. Patil* and Matthias Althoff \\ Virginia Polytechnic Institute and State University, Blacksburg, Virginia 24061-0203
}

September 7, 2010

\begin{abstract}
The paper presents a Galerkin approach for the solution of nonlinear beam equations. The approach is energy consistent, i.e., it is shown that the weighted residual integral describes energy flow. The Galerkin approach gives accurate results with less degrees of freedom as compared to low-order finite element formulation. The Galerkin approach also leads to a nonlinear order-reduction technique which can be used to further decrease the order of the system. The reduced-order model is shown to capture the dominant nonlinearities in the system and is ideal for preliminary design and control synthesis.
\end{abstract}

Brief (running head) title: Energy-consistent Galerkin approach for nonlinear beams

Keywords: Galerkin Method, Nonlinear Dynamics, Beam Theory

\section{Introduction}

There are a number of geometrically-exact formulations for the nonlinear dynamics of beams that can be used for analysis and design [Borri \& Mantegazza(1985), Bauchau \& Kang(1993), Simo \& Vu-Quoc(1988)]. These theories are displacement-based and solution of the equations requires a finite-element formulation. The present work is based on the "fully intrinsic" formulation developed by Hodges [Hodges(1990)], which can be written in a simple matrix form with only second-degree nonlinearities. To say that these equations are intrinsic is to say that they are independent of displacement and rotation measures. Recently, a set of generalized strain-velocity compatibility relations were derived by Hodges [Hodges(2003)] which, along with the equations of motion, make up a complete set of equations that can be solved without using displacement/rotation measures for certain loading and boundary conditions.

\footnotetext{
*Associate Professor, Department of Aerospace and Ocean Engineering. mpatil@vt.edu (corresponding author)

${ }^{\dagger}$ Visiting Student, Department of Aerospace and Ocean Engineering. Currently, Postdoctoral Researcher, Department of Electrical and Computer Engineering, Carnegie Mellon University, 5000 Forbes Ave., Pittsburgh, PA 15213.
} 
The paper presents a Galerkin approach for the solution of these nonlinear beam equations. Nonlinear beam analysis is required when analyzing various structures including, helicopter blades and high-aspectratio wings, as well as beams-like nano-sensors. Aeroelastic problems can be easily handled by noting that the no-penetration aerodynamic boundary conditions requires velocity rather than displacements. Velocities are primary variables in the intrinsic formulation. The intrinsic equations have been successfully used for aeroelastic analysis of wings [Patil \& Hodges(2006)], helicopter blades [Traugott et al.(2006)], as well as complete aircraft [Chang et al.(2008)] (modeled as a set of connected beams). The present analysis improves on earlier solution techniques based on nonlinear finite element approach used in Refs. [Traugott et al.(2006)], [Patil \& Hodges(2006)], and [Chang et al.(2008)]. Furthermore, the approach leads to an nonlinear, orderreduction technique which can be applied to the Galerkin equations as well as finite-element equations.

\section{Nonlinear, Intrinsic Beam Equations}

The nonlinear, intrinsic, mixed equations for the dynamics of a general (non-uniform, twisted, curved, anisotropic) beam undergoing small strains and large deformation are given below,

$$
\begin{gathered}
F^{\prime}+(\widetilde{\mathbb{k}}+\widetilde{\kappa}) F+\mathbb{f}=\dot{P}+\widetilde{\Omega} P \\
M^{\prime}+(\widetilde{\mathbb{k}}+\widetilde{\kappa}) M+\left(\widetilde{\mathbb{e}_{1}}+\widetilde{\gamma}\right) F+\mathrm{m}=\dot{H}+\widetilde{\Omega} H+\widetilde{V} P \\
V^{\prime}+(\widetilde{\mathbb{k}}+\widetilde{\kappa}) V+\left(\widetilde{\mathbb{e}_{1}}+\widetilde{\gamma}\right) \Omega=\dot{\gamma} \\
\Omega^{\prime}+(\widetilde{\mathbb{k}}+\widetilde{\kappa}) \Omega=\dot{\kappa}
\end{gathered}
$$

where ()$^{\prime}$ denotes the derivative with respect to the undeformed beam reference line and $(\dot{)})$ denotes the absolute time derivative. $F(x, t)$ and $M(x, t)$ are the measure numbers of the internal force and moment vector (generalized forces), $P(x, t)$ and $H(x, t)$ are the measure numbers of the linear and angular momentum vector (generalized momenta), $\gamma(x, t)$ and $\kappa(x, t)$ are the beam strains and curvatures (generalized strains), $V(x, t)$ and $\Omega(x, t)$ are the linear and angular velocity measures (generalized velocities), and $\mathbb{E}(x, t)$ and $\mathrm{m}(x, t)$ are the external force and moment measures. All measure numbers are calculated in the deformed cross-sectional frame. $\mathbb{k}(x)=\left\lfloor\begin{array}{lll}\mathbb{k}_{1}(x) & \mathbb{k}_{2}(x) \mathbb{k}_{3}(x)\end{array}\right\rfloor$ is the initial twist/curvature of the beam, $\mathbb{e}_{1}=\left\lfloor\begin{array}{lll}1 & 0 & 0\end{array}\right\rfloor^{T}$.

The first two equations in the above set are the equations of motion [Hodges(1990)] while the latter two are the intrinsic kinematical equations [Hodges(2003)] derived from the generalized strain-displacement and generalized velocity-displacement equations.

The generalized forces are related to the generalized strains via the cross-sectional beam stiffnesses/flexibilities. These cross-sectional properties can be calculated using an analytical thin-walled theory [Johnson et al.(2001)] or computational FEM analysis [Cesnik \& Hodges(1997)] for general configuration. Such an analysis gives the following, linear constitutive law,

$$
\left\{\begin{array}{l}
\gamma \\
\kappa
\end{array}\right\}=\left[\begin{array}{cc}
\mathbb{R} & \mathbb{S} \\
\mathbb{S}^{T} & \mathbb{T}
\end{array}\right]\left\{\begin{array}{l}
F \\
M
\end{array}\right\}
$$

where, $\mathbb{R}(x), \mathbb{S}(x), \mathbb{T}(x)$, are the cross-sectional flexibilities of the beam. Being linear, the present material law is only valid for small local strains, which can, however, lead to large global deformations.

The generalized momenta are related to the generalized velocities via the cross-sectional beam inertia,

$$
\left\{\begin{array}{l}
P \\
H
\end{array}\right\}=\left[\begin{array}{cc}
\mu \Delta & -\mu \widetilde{\xi} \\
\mu \widetilde{\xi} & I
\end{array}\right]\left\{\begin{array}{l}
V \\
\Omega
\end{array}\right\}=\left[\begin{array}{cc}
\mathbb{G} & \mathbb{K} \\
\mathbb{K} T & \mathbb{0}
\end{array}\right]\left\{\begin{array}{l}
V \\
\Omega
\end{array}\right\}
$$


where, $\mu(x), \xi(x), I(x)$ are the mass per unit length, mass center offset (vector in the cross-section from the beam reference axis to the cross-sectional mass center), and mass moment of inertia per unit length respectively.

Usually, the constitutive laws are used to replace some of the variable in terms of other. Here we decided to replace the generalized strains in terms of generalized forces (thus allowing easy specification of zero flexibility) and to replace the generalized momenta by generalized speeds (thus allowing easy specification of zero inertia).

Finally the boundary conditions need to be specified. For the given beam of length $\mathbb{L}$, there will be two boundary conditions at each end:

$$
\begin{array}{ccc}
V(\mathbb{0}, t)=\mathbb{V}^{\mathbb{D}} & \text { OR } & F(\mathbb{0}, t)=\mathbb{F}^{\mathbb{O}} \\
\Omega(\mathbb{O}, t)=\mathbb{Q}^{\mathbb{D}} & \text { OR } & M(\mathbb{O}, t)=\mathbb{M}^{\mathbb{D}} \\
V(\mathbb{L}, t)=\mathbb{V}^{\mathbb{Q}} & \text { OR } & F(\mathbb{L}, t)=\mathbb{F}^{\mathbb{E}} \\
\Omega(\mathbb{L}, t)=\mathbb{Q}^{\mathbb{L}} & \text { OR } & M(\mathbb{L}, t)=\mathbb{M}^{\mathbb{E}}
\end{array}
$$

For the formulation presented we are going to assume a cantilevered beam so as to make it easier to present. It should be noted that the formulation as well as the conclusions that would be presented are general enough to be applicable to all possible BC combinations. The BC assumed for the present analysis are:

$$
\begin{aligned}
& V(\mathbb{O}, t)=\mathbb{V}^{\mathbb{O}} \\
& \Omega(\mathbb{O}, t)=\mathbb{Q}^{\mathbb{O}} \\
& F(\mathbb{L}, t)=\mathbb{F}^{\mathbb{L}} \\
& M(\mathbb{L}, t)=\mathbb{M}^{\mathbb{L}}
\end{aligned}
$$

\section{$3 \quad$ Energy consistent weighting}

Now consider the following weighting of all the equations (equations of motion, kinematic equations as well as the boundary conditions). Note that the constitutive equations are not included as these equations are satisfied exactly.

$$
\begin{aligned}
& \int_{\mathbb{Q}}^{\mathbb{L}}\left\{V^{T}\left[\dot{P}+\widetilde{\Omega} P-F^{\prime}-(\widetilde{\mathbb{k}}+\widetilde{\kappa}) F-\mathbb{E}\right]\right. \\
& +\Omega^{T}\left[\dot{H}+\widetilde{\Omega} H+\widetilde{V} P-M^{\prime}-(\widetilde{\mathbb{k}}+\widetilde{\kappa}) M-\left(\widetilde{\mathbb{e}_{1}}+\widetilde{\gamma}\right) F-\mathrm{m}\right] \\
& +F^{T}\left[\dot{\gamma}-V^{\prime}-(\widetilde{\mathbb{k}}+\widetilde{\kappa}) V-\left(\widetilde{\mathbb{e}_{1}}+\widetilde{\gamma}\right) \Omega\right] \\
& \left.+M^{T}\left[\dot{\kappa}-\Omega^{\prime}-(\widetilde{\mathbb{k}}+\widetilde{\kappa}) \Omega\right]\right\} d x \\
& -F(\mathbb{O}, t)^{T}\left[V(\mathbb{O}, t)-\mathbb{V}^{\mathbb{D}}\right]-M(\mathbb{O}, t)^{T}\left[\Omega(\mathbb{O}, t)-\mathbb{\Omega}^{\mathbb{D}}\right] \\
& +V(\mathbb{L}, t)^{T}\left[F(\mathbb{L}, t)-\mathbb{F}^{\mathbb{L}}\right]+\Omega(\mathbb{L}, t)^{T}\left[M(\mathbb{L}, t)-\mathbb{M}^{\mathbb{L}}\right]=0
\end{aligned}
$$


Integrating by parts and then simplifying the expression we have:

$$
\begin{aligned}
\int_{\mathbb{Q}}^{\mathbb{L}}\left[V^{T} \dot{P}+\Omega^{T} \dot{H}\right] d x+\int_{\mathbb{Q}}^{\mathbb{L}}\left[F^{T} \dot{\gamma}+M^{T} \dot{\kappa}\right] d x \\
=\int_{\mathbb{Q}}^{\mathbb{L}}\left[V^{T} \mathbb{f}+\Omega^{T} \mathfrak{m}\right] d x+\left[V(\mathbb{L}, t)^{T} \mathbb{F}^{\mathbb{L}}+\Omega(\mathbb{L}, t)^{T} \mathbb{M}^{\mathbb{L}}-F(\mathbb{O}, t)^{T} \mathbb{V}^{\mathbb{Q}}-M(\mathbb{O}, t)^{T} \mathbb{R}^{\mathbb{D}}\right]=0
\end{aligned}
$$

The first term above is the rate of change of kinetic energy, the second term is the rate of change of potential energy, the third term is the rate of work done (power) due to applied forces in the interior of the beam and the fourth term is the power due to applied forces at the boundaries. The equations states that the rate of change of energy of the beam is equal to the rate of work done on the beam. Thus, the above equations is an energy balance equations. We are going to use the above equation to derive a Galerkin approach to solve the equations.

\section{Galerkin approximation}

Now let us assume that the primary variables can be written as a linear combination of a set of trial functions.

$$
\left\{\begin{array}{c}
V(x, t) \\
\Omega(x, t) \\
F(x, t) \\
M(x, t)
\end{array}\right\}=\left[\begin{array}{cccc}
V^{1}(x) & V^{2}(x) & \cdots & V^{n}(x) \\
\Omega^{1}(x) & \Omega^{2}(x) & \cdots & \Omega^{n}(x) \\
F^{1}(x) & F^{2}(x) & \cdots & F^{n}(x) \\
M^{1}(x) & M^{2}(x) & \cdots & M^{n}(x)
\end{array}\right]\left\{\begin{array}{c}
q^{1}(t) \\
q^{2}(t) \\
\vdots \\
q^{n}(t)
\end{array}\right\}
$$

The above expansion is an approximation to the exact solution and, assuming that the set of trial functions is complete, will approach the exact solution as $n \rightarrow \infty$. Now as presented above, the trial functions are coupled, i.e., a given trial function is in general comprised of 12 functions of $x$ for the 12 variables so as to keep the presentation general. One of the first sets of trial functions that is presented in the next section will be a set of independent trial functions for each variable.

The secondary variables $(P, H, \gamma, \kappa)$ are linearly related to the primary variables using the constitutive laws, which are exactly satisfied.

$$
\begin{aligned}
& \left\{\begin{array}{l}
\gamma^{i} \\
\kappa^{i}
\end{array}\right\}=\left[\begin{array}{cc}
\mathbb{R} & \mathbb{S} \\
\mathbb{S}^{T} & \mathbb{T}
\end{array}\right]\left\{\begin{array}{l}
F^{i} \\
M^{i}
\end{array}\right\} \\
& \left\{\begin{array}{l}
P^{i} \\
H^{i}
\end{array}\right\}=\left[\begin{array}{cc}
\mathbb{G} & \mathbb{K} \\
\mathbb{K}^{T} & \mathbb{\mathbb { V }}
\end{array}\right]\left\{\begin{array}{l}
V^{i} \\
\Omega^{i}
\end{array}\right\}
\end{aligned}
$$

Now we need $n$ equations which could be used to solve for the $n$ generalized coordinates. These equations come from the above energy balance equation.

$$
\begin{aligned}
& \int_{\mathbb{0}}^{\mathbb{L}}\left\{V^{j^{T}}\left[P^{i} \dot{q}^{i}+\widetilde{\Omega^{k} q^{k}} P^{i} q^{i}-F^{i^{\prime}} q^{i}-\left(\widetilde{\mathbb{k}}+\widetilde{\kappa^{k} q^{k}}\right) F^{i} q^{i}-\mathbb{E}\right]\right. \\
& +\Omega^{j^{T}}\left[H^{i} \dot{q}^{i}+\widetilde{\Omega^{k} q^{k}} H^{i} q^{i}+\widetilde{V^{k} q^{k}} P^{i} q^{i}-M^{i^{\prime}} q^{i}-\left(\widetilde{\mathbb{k}}+\widetilde{\kappa^{k} q^{k}}\right) M^{i} q^{i}-\left(\widetilde{\mathbb{E}_{1}}+\widetilde{\gamma^{k} q^{k}}\right) F^{i} q^{i}-\mathrm{m}\right] \\
& +F^{j^{T}}\left[\gamma^{i} \dot{q}^{i}-V^{i^{\prime}} q^{i}-\left(\widetilde{\mathbb{k}}+\widetilde{\kappa^{k} q^{k}}\right) V^{i} q^{i}-\left(\widetilde{\mathbb{E}_{1}}+\widetilde{\gamma^{k} q^{k}}\right) \Omega^{i} q^{i}\right] \\
& \left.+M^{j^{T}}\left[\kappa^{i} \dot{q}^{i}-\Omega^{i^{\prime}} q^{i}-\left(\widetilde{\mathbb{k}}+\widetilde{\kappa^{k} q^{k}}\right) \Omega^{i} q^{i}\right]\right\} d x \\
& -F^{j}(\mathbb{O})^{T}\left[V^{i}(\mathbb{O}) q^{i}-\mathbb{V}^{\mathbb{O}}\right]-M^{j}(\mathbb{O})^{T}\left[\Omega^{i}(\mathbb{O}) q^{i}-\mathbb{\Omega}^{\mathbb{D}}\right] \\
& +V^{j}(\mathbb{L})^{T}\left[F^{i}(\mathbb{L}) q^{i}-\mathbb{E}^{\mathbb{L}}\right]+\Omega^{j}(\mathbb{L})^{T}\left[M^{i}(\mathbb{L}) q^{i}-\mathbb{M}^{\mathbb{L}}\right]=0
\end{aligned}
$$


For each $j=1,2, \cdots, n$ we have one equation. The energy equation defined in the earlier section is a linear combination of the above equations. Thus if the $n$ equations defined above are satisfied then the energy balance equation is automatically satisfied.

\section{Independent, orthogonal polynomial trial functions}

Let all the 12 variables be represented in terms of independent generalized coordinates. Thus:

$$
\begin{aligned}
& V(x, t)=\left[\begin{array}{llll}
\Phi^{1}(x) & \Phi^{2}(x) & \cdots & \Phi^{\nu}(x)
\end{array}\right]\left\{\begin{array}{c}
v^{1}(t) \\
v^{2}(t) \\
\vdots \\
v^{\nu}(t)
\end{array}\right\} \\
& \Omega(x, t)=\left[\begin{array}{llll}
\Phi^{1}(x) & \Phi^{2}(x) & \cdots & \Phi^{\nu}(x)
\end{array}\right]\left\{\begin{array}{c}
\omega^{1}(t) \\
\omega^{2}(t) \\
\vdots \\
\omega^{\nu}(t)
\end{array}\right\} \\
& F(x, t)=\left[\begin{array}{llll}
\Phi^{1}(x) & \Phi^{2}(x) & \cdots & \Phi^{\nu}(x)
\end{array}\right]\left\{\begin{array}{c}
f^{1}(t) \\
f^{2}(t) \\
\vdots \\
f^{\nu}(t)
\end{array}\right\} \\
& M(x, t)=\left[\begin{array}{llll}
\Phi^{1}(x) & \Phi^{2}(x) & \ldots & \Phi^{\nu}(x)
\end{array}\right]\left\{\begin{array}{c}
m^{1}(t) \\
m^{2}(t) \\
\vdots \\
m^{\nu}(t)
\end{array}\right\}
\end{aligned}
$$

where,

$$
\Phi^{i}(x)=\left[\begin{array}{ccc}
\mathcal{P}^{i-1}(\bar{x}) & 0 & 0 \\
0 & \mathcal{P}^{i-1}(\bar{x}) & 0 \\
0 & 0 & \mathcal{P}^{i-1}(\bar{x})
\end{array}\right]
$$

with

$$
\bar{x}=\frac{x}{\mathbb{L}}
$$

and

$$
v^{i}(t)=\left\{\begin{array}{l}
v_{1}^{i}(t) \\
v_{2}^{i}(t) \\
v_{3}^{i}(t)
\end{array}\right\} \quad \omega^{i}(t)=\left\{\begin{array}{l}
\omega_{1}^{i}(t) \\
\omega_{2}^{i}(t) \\
\omega_{3}^{i}(t)
\end{array}\right\} \quad f^{i}(t)=\left\{\begin{array}{l}
f_{1}^{i}(t) \\
f_{2}^{i}(t) \\
f_{3}^{i}(t)
\end{array}\right\} \quad m^{i}(t)=\left\{\begin{array}{l}
m_{1}^{i}(t) \\
m_{2}^{i}(t) \\
m_{3}^{i}(t)
\end{array}\right\}
$$

The independent trial functions used are the shifted Legendre functions [Abramowitz \& Stegun(1968)]. The Legendre functions are a complete set of orthogonal polynomials. The shifted Legendre functions are orthogonal over the shifted (0 - 1) interval as shown below:

$$
\int_{0}^{1} \mathcal{P}^{i}(\bar{x}) \mathcal{P}^{j}(\bar{x}) d \bar{x}=\delta_{i j} \frac{1}{2 i+1}
$$

The polynomials can be iteratively derived as:

$$
\begin{gathered}
\mathcal{P}^{0}(\bar{x})=1 \quad \mathcal{P}^{1}(\bar{x})=2 \bar{x}-1 \\
\mathcal{P}^{i+1}(\bar{x})=\frac{(2 i+1)(2 \bar{x}-1) \mathcal{P}^{i}(\bar{x})-i \mathcal{P}^{i-1}(\bar{x})}{i+1}
\end{gathered}
$$


The Galerkin equations can be derived as:

$$
\begin{aligned}
& \int_{\mathbb{0}}^{\mathbb{L}} \Phi^{j}\left[\left(\mathbb{G} \Phi^{i} \dot{v}^{i}+\mathbb{K} \Phi^{i} \dot{\omega}^{i}\right)+\widetilde{\Phi^{k} \omega^{k}}\left(\mathbb{G} \Phi^{i} v^{i}+\mathbb{K} \Phi^{i} \omega^{i}\right)-\Phi^{i^{\prime}} f^{i}-\left(\widetilde{\mathbb{k}}+\widetilde{\mathbb{S}^{T} \Phi^{k} f^{k}}+\widetilde{\mathbb{T} \Phi^{k} m^{k}}\right) \Phi^{i} f^{i}-\mathbb{E}\right] d x \\
& +\Phi^{j}(\mathbb{L})\left[\Phi^{i}(\mathbb{L}) f^{i}-\mathbb{F}^{\mathbb{L}}\right]=0 \\
& \int_{\mathbb{0}}^{\mathbb{L}} \Phi^{j}\left[\left(\mathbb{K}^{T} \Phi^{i} \dot{v}^{i}+\square \Phi^{i} \dot{\omega}^{i}\right)+\widetilde{\Phi^{k} \omega^{k}}\left(\mathbb{K}^{T} \Phi^{i} v^{i}+\square \Phi^{i} \omega^{i}\right)+\widetilde{\Phi^{k} v^{k}}\left(\mathbb{G} \Phi^{i} v^{i}+\mathbb{K} \Phi^{i} \omega^{i}\right)-\Phi^{i^{\prime}} m^{i}\right. \\
& \left.-\left(\widetilde{\mathbb{k}}+\widetilde{\mathbb{S}^{T} \Phi^{k} f^{k}}+\widetilde{\mathbb{T} \Phi^{k} m^{k}}\right) \Phi^{i} m^{i}-\left(\widetilde{\mathbb{E}_{1}}+\widetilde{\mathbb{R} \Phi^{k} f^{k}}+\widetilde{\mathscr{S} \Phi^{k} m^{k}}\right) \Phi^{i} f^{i}-\mathbf{m}\right] d x+\Phi^{j}(\mathbb{L})\left[\Phi^{i}(\mathbb{L}) m^{i}-\mathbb{M}^{\mathbb{Q}}\right]=0 \\
& \int_{\mathbb{0}}^{\mathbb{L}} \Phi^{j}\left[\left(\mathbb{R} \Phi^{k} \dot{f}^{k}+\mathbb{S} \Phi^{k} \dot{m}^{k}\right)-\Phi^{i^{\prime}} v^{i}-\left(\widetilde{\mathbb{k}}+\widetilde{\mathbb{S}^{T} \Phi^{k} f^{k}}+\widetilde{\mathbb{T} \Phi^{k} m^{k}}\right) \Phi^{i} v^{i}-\left(\widetilde{\mathbb{E}_{1}}+\widetilde{\mathbb{R} \Phi^{k} f^{k}}+\widetilde{\mathbb{S} \Phi^{k} m^{k}}\right) \Phi^{i} \omega^{i}\right] d x \\
& -\Phi^{j}(\mathbb{0})\left[\Phi^{i}(\mathbb{0}) v^{i}-V^{\mathbb{0}}\right]=0 \\
& \int_{\mathbb{Q}}^{\mathbb{L}} \Phi^{j}\left[\left(\mathbb{S}^{T} \Phi^{k} \dot{f}^{k}+\mathbb{\mathbb { T }} \Phi^{k} \dot{m}^{k}\right)-\Phi^{i^{\prime}} \omega^{i}-\left(\widetilde{\mathbb{k}}+\widetilde{\mathbb{S}^{T} \Phi^{k} f^{k}}+\widetilde{\mathbb{T} \Phi^{k} m^{k}}\right) \Phi^{i} \omega^{i}\right] d x-\Phi^{j}(\mathbb{O})\left[\Phi^{i}(\mathbb{O}) \omega^{i}-\hat{\mathbb{\Omega}}^{\mathbb{0}}\right]=0
\end{aligned}
$$

where, $i, j, k=1,2, \cdots, \nu$. with $n=12 \times \nu$, the generalized coordinates can be represented as:

$$
\left\{\begin{array}{c}
q^{1}(t) \\
q^{2}(t) \\
\vdots \\
q^{n}(t)
\end{array}\right\}=\left\{\begin{array}{c}
v^{1}(t) \\
\omega^{1}(t) \\
f^{1}(t) \\
m^{1}(t) \\
\vdots \\
\vdots \\
v^{\nu}(t) \\
\omega^{\nu}(t) \\
f^{\nu}(t) \\
m^{\nu}(t)
\end{array}\right\}
$$

\section{Special case: constant cross-section, curvature and loading}

To obtain the above equations in a form suitable for solution, we need to calculate the integrals. For demonstration, we will assume a constant cross-section $(\mathbb{R}, \mathbb{S}, \mathbb{T}, \mathbb{G}, \mathbb{K}, \mathbb{\mathbb { V }}$ are all constants), constant curvature ( $\mathbb{k}$ is constant) and constant distributed loading ( $\mathbb{F}, \mathfrak{m}$ are constants). Furthermore we will assume that the

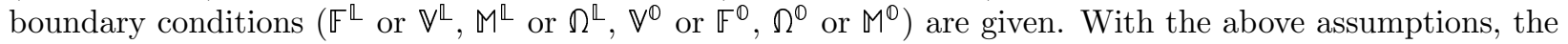


equations become:

$$
\begin{aligned}
& \mathcal{A}^{j i} \mathbb{\complement}\left(\mathbb{G} \dot{v}^{i}+\mathbb{K} \dot{\omega}^{i}\right)+\mathcal{C}^{j i k} \mathbb{\complement} \widetilde{\omega^{k}}\left(\mathbb{G} v^{i}+\mathbb{K} \omega^{i}\right)-\mathcal{B}^{j i} f^{i} \\
& -\mathcal{A}^{j i} \mathbb{\complement} \widetilde{k} f^{i}-\mathcal{C}^{j i k} \mathbb{\complement}\left(\widetilde{\mathbb{S}^{T} f^{k}}+\widetilde{\mathbb{T} m^{k}}\right) f^{i}-\mathcal{D}^{j} \mathbb{L} \mathfrak{F}+\mathcal{P}^{j}(1) \mathcal{P}^{i}(1) f^{i}-\mathcal{P}^{j}(1) \mathbb{F}^{\mathbb{L}}=0 \\
& \mathcal{A}^{j i} \mathbb{L}\left(\mathbb{K}^{T} \dot{v}^{i}+\square \dot{\omega}^{i}\right)+\mathcal{C}^{j i k} \mathbb{\complement} \widetilde{\omega^{k}}\left(\mathbb{K}^{T} v^{i}+\square \omega^{i}\right)+\mathcal{C}^{j i k} \mathbb{L} \widetilde{v^{k}}\left(\mathbb{G} v^{i}+\mathbb{K} \omega^{i}\right) \\
& -\mathcal{B}^{j i} m^{i}-\mathcal{A}_{j i} \mathbb{\mathbb { L }} \widetilde{\mathbb{k}} m^{i}-\mathcal{C}^{j i k} \mathbb{L}\left(\widetilde{\mathbb{S}^{T} f^{k}}+\widetilde{\mathbb{\mathbb { m }} m^{k}}\right) m^{i}-\mathcal{A}^{j i} \mathbb{L} \widetilde{\mathbb{e}_{1}} f^{i} \\
& -\mathcal{C}^{j i k} \mathbb{L}\left(\widetilde{\mathbb{R} f^{k}}+\widetilde{\mathbb{S m}^{k}}\right) f^{i}-\mathcal{D}^{j} \mathbb{L m}+\mathcal{P}^{j}(1) \mathcal{P}^{i}(1) m^{i}-\mathcal{P}^{j}(1) \mathbb{M}^{\mathbb{L}}=0 \\
& \mathcal{A}^{j i} \mathbb{L}\left(\mathbb{R} \dot{f}^{k}+\mathbb{S} \dot{m}^{k}\right)-\mathcal{B}^{j i} v^{i}-\mathcal{A}^{j i} \mathbb{\mathbb { k }} v^{i}-\mathcal{C}^{j i k} \mathbb{L}\left(\widetilde{\mathbb{S}^{T} f^{k}}+\widetilde{\mathbb{\mathbb { m }} m^{k}}\right) v^{i} \\
& -\mathcal{A}^{j i} \mathbb{\complement} \widetilde{\mathbb{E}_{1}} \omega^{i}-\mathcal{C}^{j i k} \llbracket\left(\widetilde{\mathbb{R} f^{k}}+\widetilde{\mathbb{S}^{k}}\right) \omega^{i}-\mathcal{P}^{j}(0) \mathcal{P}^{i}(0) v^{i}+\mathcal{P}^{j}(0) \mathbb{V}^{\mathbb{D}}=0 \\
& \mathcal{A}^{j i} \mathbb{L}\left(\mathbb{S}^{T} \dot{f}^{k}+\mathbb{\mathbb { m }} \dot{m}^{k}\right)-\mathcal{B}^{j i} \omega^{i}-\mathcal{A}^{j i} \mathbb{\mathbb { R }} \widetilde{k} \omega^{i}-\mathcal{C}^{j i k} \mathbb{\mathbb { L }}\left(\widetilde{\mathbb{S}^{T} f^{k}}+\widetilde{\mathbb{T} m^{k}}\right) \omega^{i} d x \\
& -\mathcal{P}^{j}(0) \mathcal{P}^{i}(0) \omega^{i}+\mathcal{P}^{j}(0) \Omega^{\mathbb{D}}=0
\end{aligned}
$$

Summation is implied over indices $i$ and $k$. And $\mathcal{A}^{j i}, \mathcal{B}^{j i}, \mathcal{C}^{j i k}$ and $\mathcal{D}^{j}$ are non-dimensional integrals as given below:

$$
\begin{gathered}
\mathcal{A}^{j i}=\int_{0}^{1} \mathcal{P}^{j}(\bar{x}) \mathcal{P}^{i}(\bar{x}) d \bar{x} \\
\mathcal{B}^{j i}=\int_{0}^{1} \mathcal{P}^{j}(\bar{x})\left(\mathcal{P}^{i}(\bar{x})\right)^{\prime} d \bar{x} \\
\mathcal{C}^{j i k}=\int_{0}^{1} \mathcal{P}^{j}(\bar{x}) \mathcal{P}^{i}(\bar{x}) \mathcal{P}^{k}(\bar{x}) d \bar{x} \\
\mathcal{D}^{j}=\int_{0}^{1} \mathcal{P}^{j}(\bar{x}) d \bar{x}
\end{gathered}
$$

The above set of equations can be written in the following form:

$$
A_{j i} \dot{q}_{i}+B_{j i} q_{i}+C_{j i k} q_{i} q_{k}+D_{j}=0
$$

\section{Solution}

Given the above set of nonlinear equations for the dynamics of beam, we can have the following solutions:

- Nonlinear steady-state solution

- Linear dynamic perturbation solution about the nonlinear steady state

- Nonlinear dynamic solution 


\subsection{Nonlinear steady-state solution}

The linear steady state solution is calculated by solving:

$$
B_{j i} \bar{q}_{i}+C_{j i k} \bar{q}_{i} \bar{q}_{k}+\bar{D}_{j}=0
$$

where, $\bar{q}$ refers to steady-state solution due to steady-state forcing calculated in $\bar{D}$. The solution is calculated using Newton-Raphson iterations. The Jacobian required for the iteration can be easily calculated as:

$$
\mathcal{J}(q)=B_{j i}+C_{j i k} q_{k}+C_{j k i} q_{k}
$$

Since the highest order of nonlinearity is second, the Newton-Raphson method leads to converged solution.

\subsection{Nonlinear equations at the steady-state}

Now, let the total applied force be given by:

$$
D=\bar{D}+\widehat{D}(t)
$$

and the corresponding solution be:

$$
q=\bar{q}+\widehat{q}(t)
$$

Substituting it in the equations of motion we have the equations with respect to the steady-state solution as:

$$
\widehat{A}_{j i} \dot{\hat{q}}_{i}+\widehat{B}_{j i} \widehat{q}_{i}+\widehat{C}_{j i k} \widehat{q}_{i} \widehat{q}_{k}+\widehat{D}_{j}=0
$$

where,

$$
\begin{gathered}
\widehat{A}_{j i}=A_{j i} \\
\widehat{B}_{j i}=B_{j i}+C_{j i k} \bar{q}_{k}+C_{j k i} \bar{q}_{k} \\
\widehat{C}_{j i k}=C_{j i k}
\end{gathered}
$$

The above equation are completely nonlinear with the origin at the steady-state.

\subsection{Linearized equations at the steady-state}

The linearized system is given by:

$$
\widehat{A}_{j i} \dot{\widehat{q}}_{i}+\widehat{B}_{j i} \widehat{q}_{i}+\widehat{D}_{j}=0
$$

The linear modes at the steady-state are calculated using the linearized eigenvalue problem at the steady-state:

$$
\widehat{A}_{j i} \dot{\widehat{q}}_{i}+\widehat{B}_{j i} \widehat{q}_{i}=0
$$




\subsection{Nonlinear dynamic simulation}

Simulation of the complete nonlinear dynamic system requires a time marching algorithm applied to the complete set of nonlinear equations given in Eq. 45. Here we use the central difference algorithm which converts the nonlinear differential equations to nonlinear static equations at each time step. The nonlinear equations can be solved to determine $q^{t+\Delta t}$ (the variables at the next time step) in terms of $q^{t}$ (the variables at the current time step):

$$
\left(\frac{A_{j i}}{\Delta t}+\frac{B_{j i}}{2}+\frac{\left(C_{j i k}+C_{j k i}\right) q_{k}^{t}}{4}\right) q_{i}^{t+\Delta t}+\frac{C_{j i k}}{4} q_{i}^{t+\Delta t} q_{k}^{t+\Delta t}+\left(-\frac{A_{j i}}{\Delta t} q_{i}^{t}+\frac{B_{j i}}{2} q_{i}^{t}+\frac{C_{j i k}}{4} q_{i}^{t} q_{k}^{t}+\frac{D_{j}^{t}+D_{j}^{t+\Delta t}}{2}\right)=0
$$

Note that the above equations have exactly the same form as the nonlinear equations for steady state calculations (Eq. 46). Thus, the time marching is conducted by solving the nonlinear equation using NewtonRaphson at each step.

\section{Modal transformation and reduction}

Now consider representing the variables in terms of the modal co-ordinates:

$$
q_{i}=U_{i l} \xi_{l}
$$

where, $U_{i 1}, U_{i 2}, \cdots$ are the modes of linearized system (Eq. 55). If the length of $\xi$ is less then the length of $q$ then we have reduction in the number of states of the system. The reduced order system can be written as:

$$
A_{m l}^{r} \dot{\xi}_{l}+B_{m l}^{r} \xi_{l}+C_{m l n}^{r} \xi_{l} \xi_{n}+D_{m}^{r}=0
$$

where,

$$
\begin{gathered}
A_{m l}^{r}=A_{j i} U_{j m} U_{i l} \\
B_{m l}^{r}=B_{j i} U_{j m} U_{i l} \\
C_{m l n}^{r}=C_{j i k} U_{j m} U_{i l} U_{k n} \\
D_{m}^{r}=D_{j} U_{j m}
\end{gathered}
$$

There are two points to be noted. Firstly, the simple form of the modal transformation equation (Eq. 62) is possible due to the specific order of the equations, i.e., the equations are ordered so that the corresponding weighing functions are in the same order as the variables in the generalized coordinate vector.

Secondly, it should be noted that the system equations are in state-space form. Thus, the eigenvalues and eigenvectors of the system will be, in general, complex. Each vibration mode will be represented by two eigenvalues which are complex conjugates and the corresponding eigenvectors which are also complex conjugates. To avoid complex modal coordinates the pair of complex conjugate eigenvectors is represented by two real vectors made up of the real and imaginary parts [Patil(2000)]. 


\section{Results}

The nonlinear Galerkin approach was applied to a simple prismatic beam case presented in Table 1. The approach is validated by solving a simple steady nonlinear beam problem which has an exact solution. This is followed by perturbation analysis to detemine the structural frequencies (linear as well as linearized about a nonlinear steady state). Then results are presented that illustrate the effectiveness of the nonlinear reduced order modeling approach. Finally, nonlinear simulation is conducted to show the energy conservation of the present formulation.

\subsection{Nonlinear steady state solution}

Let us first consider a beam with tip follower force. As the force increases, the deformation increases; and due to the large deformation, the force direction as well as its position relative to the beam root changes. The correct nonlinear solution thus has a lower root bending moment as compared to linear solution. The exact solution to the problem satisfies the following transcendental equation:

$$
\frac{1}{6} \bar{m} \sqrt{4-\bar{m}^{4} \bar{p}^{2}}+\frac{2 \sqrt{2}}{3 \sqrt{\bar{p}}} F\left[\sin ^{-1}\left(\frac{\bar{m} \sqrt{\bar{p}}}{\sqrt{2}}\right) \mid-1\right]=1-\bar{x}
$$

where $\bar{m}$ is the bending moment made dimensionless by the linear bending moment $(P L), \bar{p}$ is the tip force made dimensionless by the bending rigidity and beam length $\left(E I / L^{2}\right), \bar{x}$ is the axial coordinate made dimensionless by beam length $(L)$, and $F(\phi \mid k)$ is the elliptic integral of the first kind with $k=-1$ and $\sin \phi=\frac{\bar{m} \sqrt{\bar{p}}}{\sqrt{2}}$.

Figure 1 presents the result for $\bar{p}=3$. Since the problem is nonlinear, we see in Figure 1(a) that the bending moment distribution is not linear. For $n=3$ we get result close to the exact result. The solution is the best solution considering that the variable are allowed to vary only quadratically over the domain. Because the boundary conditions are weakly satisfied, the present solution approach weighs the error in the boundary and interior in an energy consistent way. For $n=6$ the results are right on top of the exact results for the domain as well as the boundary. Figure 1(b) shows the convergence of the solution by plotting the reduction in error with increasing order of the approximation. The exact solution for the root bending moment for $\bar{p}=3$ is $\bar{m}(0)=-0.81044$. The figure shows the absolute difference between the exact and approximate solution for the root bending moment and shows the absolute value of the bending moment at the tip (which should be zero for exact satisfaction of boundary conditions). The error reduced with increase in order of the approximation and the root bending moment reaches the exact solution (using double precision) after around 12 polynomials per variable.

\subsection{Linearized perturbation analysis}

Next we conduct perturbation analysis to calculate the natural frequencies of the beam. Table 2 lists the calculated frequencies and compares the results with exact results [Wright et al.(1982)] as well as FEM solution [Traugott et al.(2006)]. The frequency predictions of a non-rotating beam are linear results. For the rotating beam, one has to calculate the nonlinear steady state results, then perturb and linearize the problem to determine the frequencies. For all the cases presented, the present Galerkin approach with 10 assumed modes per variable (120 states) are exact to three significant digits. On the other hand, FEM solution with 10 nodes (120 states), is not very accurate leading to errors greater than $10 \%$ for the third bending mode. 
Fig 2 shows the convergence of the Galerkin approach and compares it to the convergence of the FEM approach. The Galerkin approximation is very accurate and the error decreases sharply with increasing modes. Using around 8 trial functions per variable (less than 100 total variables), we reach the minimum error limit for double precision calculations. The FEM approach has a smooth second-order convergence which means one would require over a million finite elements to get to the error limit.

Not yet addressed in the present study is the impact of bandedness or sparsity of the matrices. For low-order finite elements, the coefficient matrices are very sparse, and though we have not yet done so, one can take advantage of this. Also, the coefficient matrices for low-order elements can be calculated using lower-order Gauss integration thus further reducing the computational time. A Galerkin approach applied to general configurations requires calculation of complex integrals and will lead to fully populated matrices. Thus, the Galerkin approach, though more accurate, may not be computationally as efficient as the highorder elements for general configurations with discontinuities. But, if one is interested in using the nonlinear beam analysis for preliminary design or control synthesis, the reduced number of degrees of freedom are essential.

\subsection{Reduced-order modeling}

The present approach can be used to further reduce the order of the system by using the nonlinear modal reduction approach. Fig 3(a) shows the change in frequency of the beam with applied vertical load. The beam deforms due to the load and the large deformation leads to coupling between the horizontal bending and torsion modes. This is a nonlinear coupling and can be captured by a model with correct nonlinear terms. We retained 4 vertical bending, 2 torsion and 2 horizontal bending modes of the unloaded model in the reduced order model. Thus, the frequencies of the linearized system is exact for no load. As we load the beam the deformation induces nonlinear coupling which is captured by the reduced order model.

Fig 3(b) shows the results of a reduced-order model for a rotating beam. Again, the modes of the system without transverse loads are used to model the system with loads. As in the non-rotating case, the reducedorder model is able to capture the nonlinear torsion-horizontal-bending coupling. One could improve on these results by adding perturbation modes [Bauchau \& Guernsey(1993)] or with nonlinear normal modes analysis [Shaw \& Pierre(1992)].

\subsection{Nonlinear dynamics}

Lets consider a case wherein the beam is initially statically deformed using a distributed load in the two bending directions of $f_{y}=f_{z}=10 \mathrm{~N} / \mathrm{m}$. This leads to significant deformation in the vertical direction but equivalent loads in the two directions. This deformation is used as the initial condition for nonlinear simulation of the beam. Results are presented for three cases: $n=3$ where all the variables are essentially approximated using quadratic polynomials leading to a total of 36 degrees of freedom (dof), $n=9$ which leads to 108 dof, and ROM which is a reduced order model of the $n=9$ case by using the first 18 modes leading to 36 dof. For all practical purposes the results are converged for the $n=9$ case.

Figure 4(a) shows the vertical velocity as a function of time. It can be seen that the $n=3$ case does not lead to accurate results as expected but still provides a good estimate. On the other hand, the ROM results are very close to the converged results even though it uses only 36 dof. 
Figure 4(b) shows the bending moment due to horizontal motion at the root. As expected we see higher frequencies due to the higher horizontal bending stiffness. Again the results for ROM are very close to $n=9$ while the $n=3$ results give a good approximation.

Figure 4(c) shows the twisting moment at the root. Twisting moment is a purely nonlinear response because linear beam problem with horizontal and vertical forces does not lead to twisting moment. Even for the nonlinear part of the solution, the ROM result are very accurate and $n=3$ results are still approximate. It should be noted that a linear modal analysis results would lead to zero twisting moment. Thus, this figure illustrates the accuracy of ROM in correctly capturing the nonlinearities in a lower order model.

Figure 4(d) shows the vertical velocity at the root. Since this is a cantilevered beam, the exact result imposed weakly for the boundary condition is zero. The value of the root velocity is thus an indication of the error in the solution. We see that the $n=3$ case has significant error - of the order of 2 when the tip velocities are of the order of 10 . For $n=9$ case this error reduces to be of the order of 0.1 (around $1 \%$ ). This reduction in error is maintained in the ROM.

Figure 4(e) shows the total energy of the beam as a function of time. Since we have a case of nonlinear free vibration, we do not expect any change in the energy, and as can be seen the energy remains constant. The energy for each case if different because each case leads to a slightly different initial condition, but the results are still within 3\% of each other. Figure 4(f) shows the change in energy relative to the energy at the initial condition. The change in energy is of the order of $10^{-13}$ while the actual energy is of the order of 10. This is the case even for the nonlinear reduced order model. Thus due to energy consistent weighting, the energy of the beam remains constant through nonlinear dynamic simulation.

\section{Conclusions}

The paper presents a Galerkin approach for solving the dynamic, nonlinear, beam equations. The solution approach gives very accurate results using low number of degrees of freedom. As expected, the Galerkin approach leads to considerable increase in the accuracy as compared to low-order finite element formulation. It was noted that for a general case, the upfront computational requirements of the Galerkin method would be high due to the calculation of complex integrals. The Galerkin methodology leads to a nonlinear orderreduction technique which can further decrease the number of degrees of freedom in the problem. The reduced order model based on linear (and thus uncoupled) modes is able to capture the nonlinear torsion-horizontalbending coupling of a beam during large deformation. This was shown using linearized perturbation analysis

as well as nonlinear simulation. Thus, the reduced-order model is able to capture the dominant nonlinearities in the system and would be ideal for aeroelasticity, preliminary design, and control synthesis.

\section{References}

[Abramowitz \& Stegun(1968)] Abramowitz, M. \& Stegun, I. A. (1968). Handbook of Mathematical Functions. New York: Dover Publications.

[Bauchau \& Guernsey(1993)] Bauchau, O. A. \& Guernsey, D. (1993). On the choice of appropriate bases for nonlinear dynamic modal analysis. Journal of the American Helicopter Society, 38(4), 28 - 36.

[Bauchau \& Kang(1993)] Bauchau, O. A. \& Kang, N. K. (1993). A multibody formulation for helicopter structural dynamic analysis. Journal of the American Helicopter Society, 38(2), 3-14. 
[Borri \& Mantegazza(1985)] Borri, M. \& Mantegazza, P. (1985). Some contributions on structural and dynamic modeling of helicopter rotor blades. l'Aerotecnica Missili e Spazio, 64 (9), 143 - 154.

[Cesnik \& Hodges(1997)] Cesnik, C. E. S. \& Hodges, D. H. (1997). VABS: a new concept for composite rotor blade cross-sectional modeling. Journal of the American Helicopter Society, 42(1), 27 - 38.

[Chang et al.(2008)] Chang, C.-S., Hodges, D. H., \& Patil, M. J. (2008). Flight dynamics of highly flexible aircraft. Journal of Aircraft, 45(3), 538-545.

[Hodges(1990)] Hodges, D. H. (1990). A mixed variational formulation based on exact intrinsic equations for dynamics of moving beams. International Journal of Solids and Structures, 26(11), 1253 - 1273.

[Hodges(2003)] Hodges, D. H. (2003). Geometrically exact, intrinsic theory for dynamics of curved and twisted anisotropic beams. AIAA Journal, 41(6), 1131-1137.

[Johnson et al.(2001)] Johnson, E. R., Vasiliev, V. V., \& Vasiliev, D. V. (2001). Anisotropic thin-walled beams with closed cross-sectional contours. AIAA Journal, 39(12), 2389-2393.

[Patil(2000)] Patil, M. J. (2000). Decoupled second-order equations and modal analysis of a general nonconservative system. In Proceedings of the AIAA Dynamics Specialists Conference. Atlanta, Georgia. AIAA-2000-1654.

[Patil \& Hodges(2006)] Patil, M. J. \& Hodges, D. H. (2006). Flight dynamics of highly flexible flying wings. Journal of Aircraft, 43(6), 1790-1798.

[Shaw \& Pierre(1992)] Shaw, S. W. \& Pierre, C. (1992). Normal modes for nonlinear vibratory systems. Journal of Sound and Vibration, 164(1), 85 - 124.

[Simo \& Vu-Quoc(1988)] Simo, J. C. \& Vu-Quoc, L. (1988). On the dynamics in space of rods undergoing large motions - a geometrically exact approach. Computer Methods in Applied Mechanics and Engineering, 66, 125-161. North Holland.

[Traugott et al.(2006)] Traugott, J. P., Patil, M. J., \& Holzapfel, F. (2006). Nonlinear dynamics and control of integrally actuated helicopter blades. Aerospace Science and Technology, 10, $509-518$.

[Wright et al.(1982)] Wright, A. D., Smith, C. E., Thresher, R. W., \& Wang, J. L. C. (1982). Vibration modes of centrifugally stiffened beams. Journal of Applied Mechanics, 49, $197-202$. 
Table 1: Beam data (for model validation purpose)

\begin{tabular}{|r|l|}
\hline \hline Span & $16 \mathrm{~m}$ \\
Chord & $1 \mathrm{~m}$ \\
Mass per unit length & $0.75 \mathrm{~kg} / \mathrm{m}$ \\
Mom. Inertia (50\% chord) & $0.1 \mathrm{~kg} \mathrm{~m}$ \\
Spanwise elastic axis & $50 \%$ chord \\
Center of gravity & $50 \%$ chord \\
Bending rigidity & $2 \times 10^{4} \mathrm{~N} \mathrm{~m}^{2}$ \\
Torsional rigidity & $1 \times 10^{4} \mathrm{~N} \mathrm{~m}^{2}$ \\
Bending rigidity (chordwise) & $4 \times 10^{6} \mathrm{~N} \mathrm{~m}^{2}$ \\
Shear/Extensional rigidity & $\infty$ \\
\hline \hline
\end{tabular}

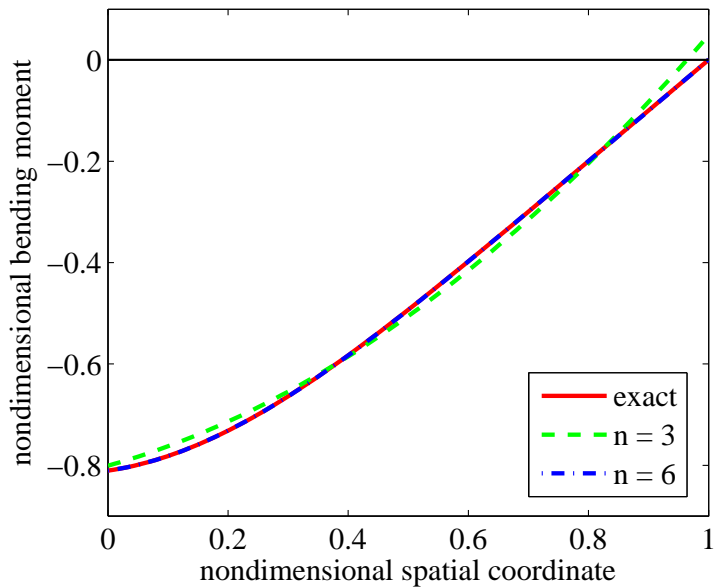

(a)

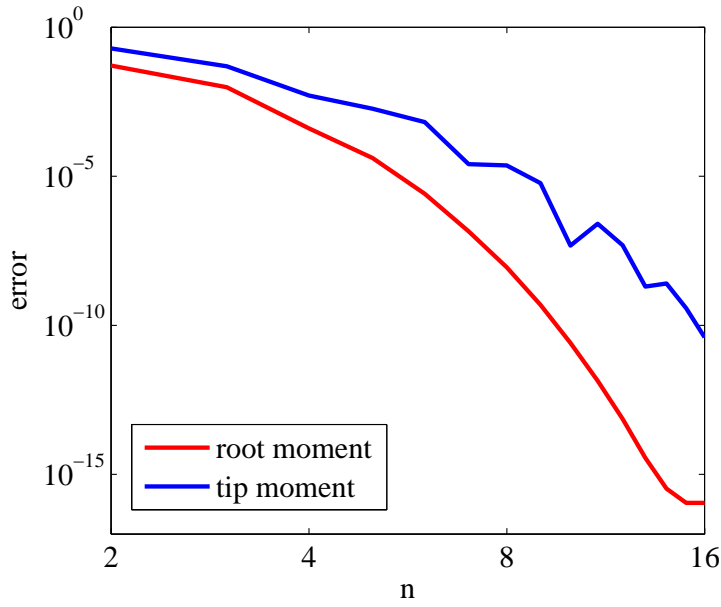

(b)

Figure 1: solution for a follower force at the tip $(\bar{p}=3):(a)$ bending moment distribution; (b) convergence of result 
Table 2: Beam structural frequencies (for model validation purpose)

\begin{tabular}{|l|ccc|}
\hline \hline Mode (rad/s) & Exact & $\begin{array}{c}\text { Present } \\
10 \text { modes }\end{array}$ & $\begin{array}{c}\text { FEM } \\
10 \text { nodes }\end{array}$ \\
\hline \multicolumn{4}{|c|}{ Cantilevered Blade: $\omega=0 \& v=0$} \\
\hline $1^{\text {st }}$ bending & 2.243 & 2.243 & 2.252 \\
$2^{\text {nd }}$ bending & 14.06 & 14.06 & 14.74 \\
$3^{\text {rd }}$ bending & 39.36 & 39.36 & 44.94 \\
$1^{\text {st }}$ torsion & 31.05 & 31.05 & 31.12 \\
$2^{\text {nd }}$ torsion & 93.14 & 93.14 & 95.32 \\
\hline \multicolumn{4}{|r|}{ Rotating Cantilevered Blade: $\omega=3.189 \mathrm{rad} / \mathrm{s} \mathrm{\&} v=0$} \\
\hline $1^{\text {st }}$ bending & 4.114 & 4.114 & 4.110 \\
$2^{\text {nd }}$ bending & 16.23 & 16.23 & 16.88 \\
$3^{\text {rd }}$ bending & 41.59 & 41.59 & 47.12 \\
\hline Rotating Cantilevered Blade with Offset: $\omega=3.189 \mathrm{rad} / \mathrm{s} \mathrm{\&} v=51.03 \mathrm{~m} / \mathrm{s}$ \\
\hline $1^{\text {st }}$ bending & 5.703 & 5.703 & 5.696 \\
$2^{\text {nd }}$ bending & 18.72 & 18.72 & 19.35 \\
$3^{\text {rd }}$ bending & 44.50 & 44.50 & 49.97 \\
\hline
\end{tabular}

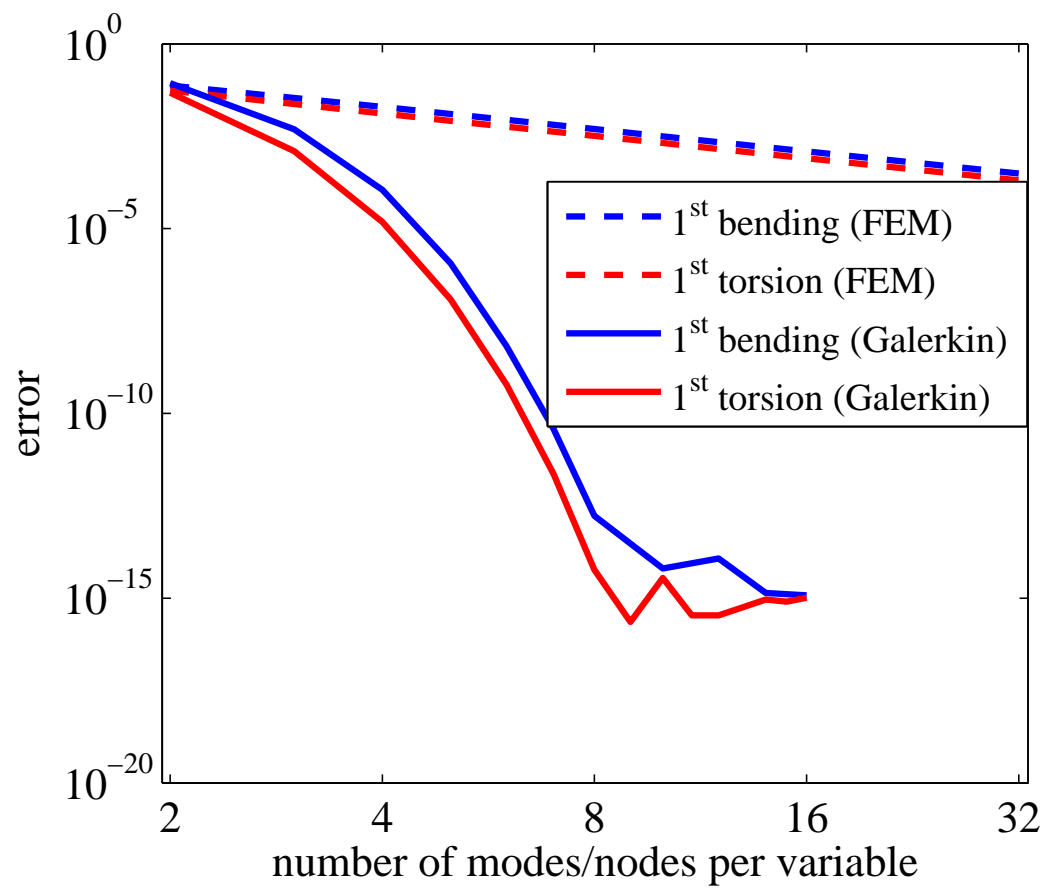

Figure 2: Accuracy of the Galerkin approach 


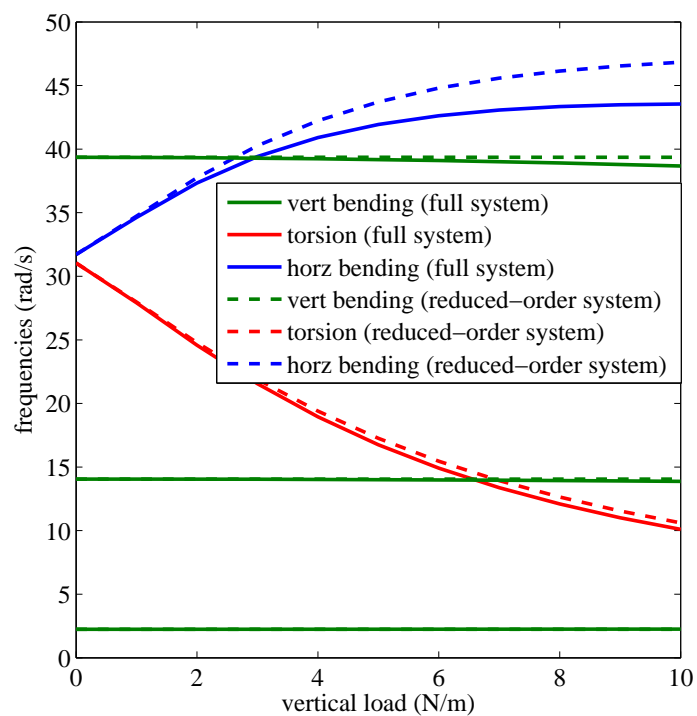

(a)

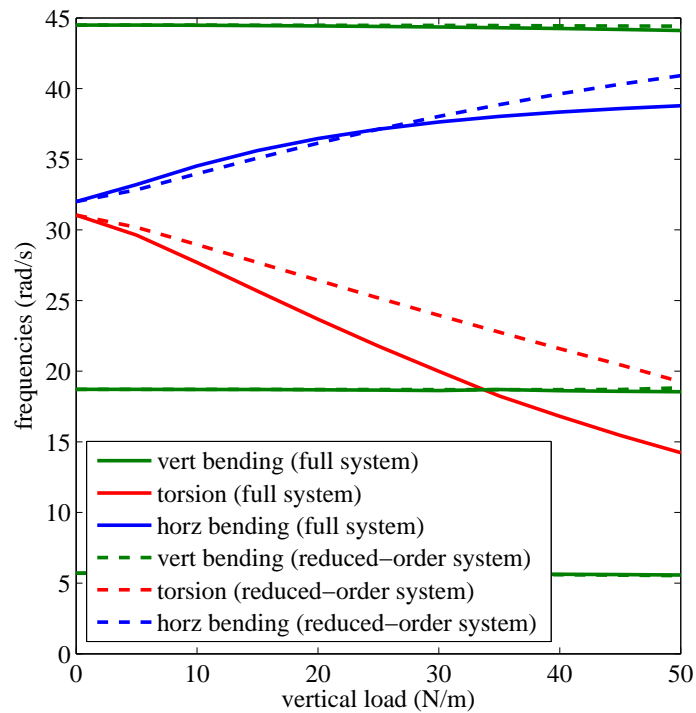

(b)

Figure 3: Efficacy of the reduced-order model in capturing nonlinearities : (a) non-rotating beam; (b) rotating beam 


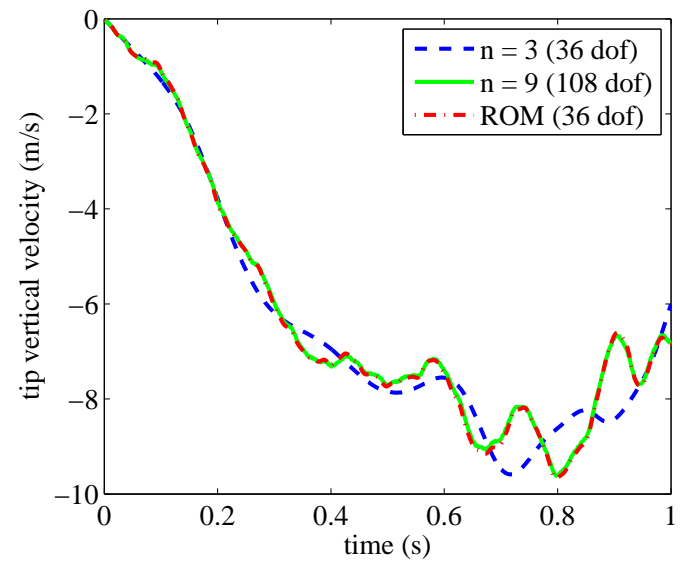

(a)

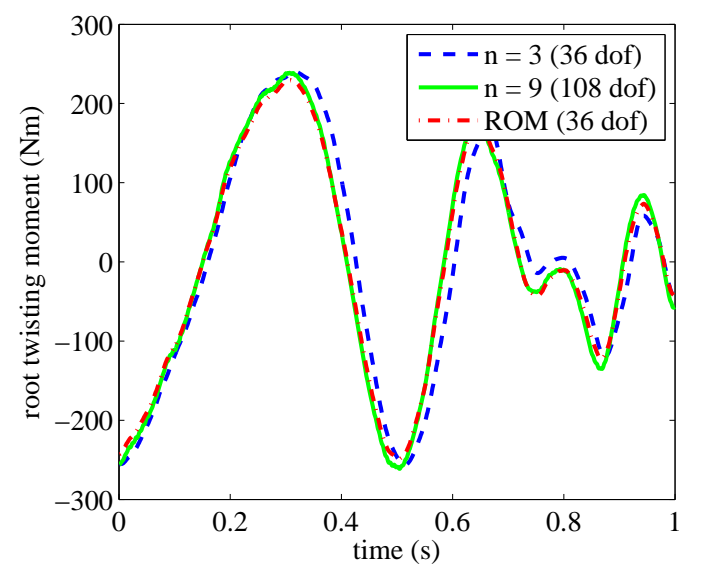

(c)

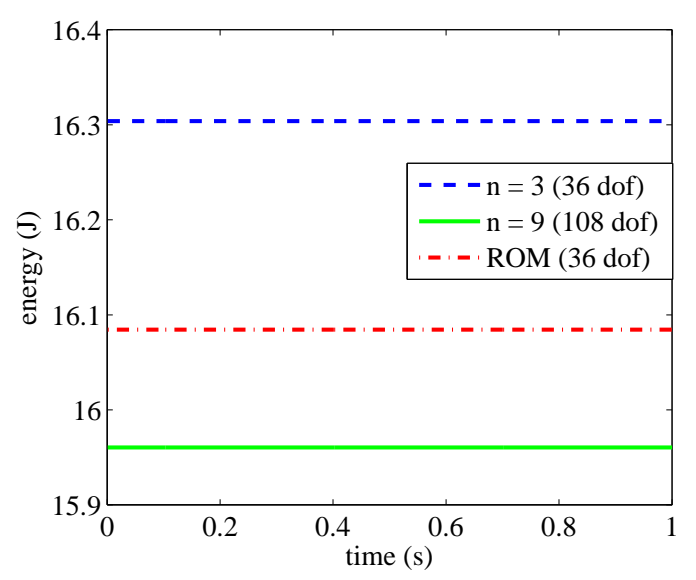

(e)

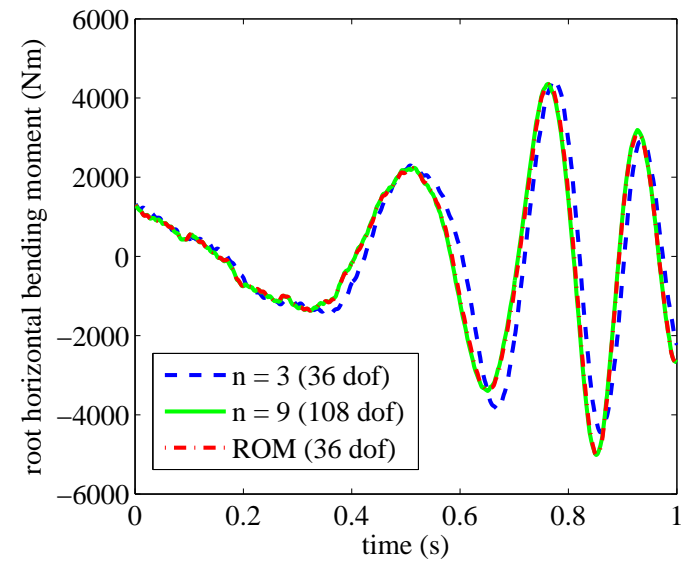

(b)

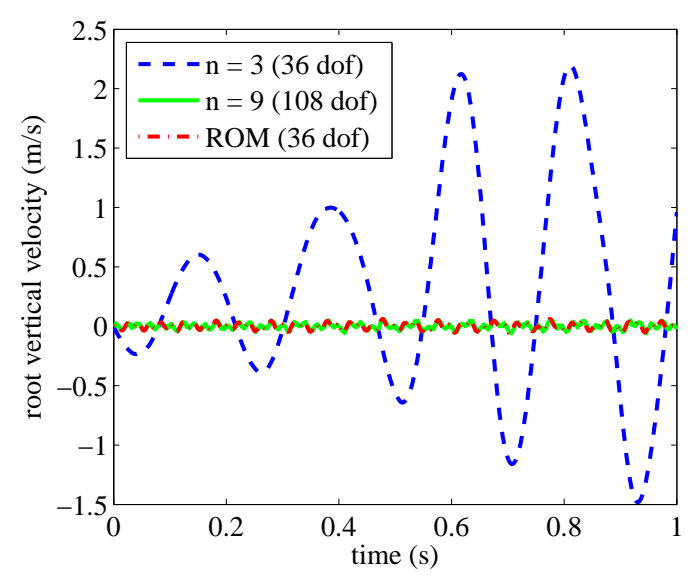

(d)

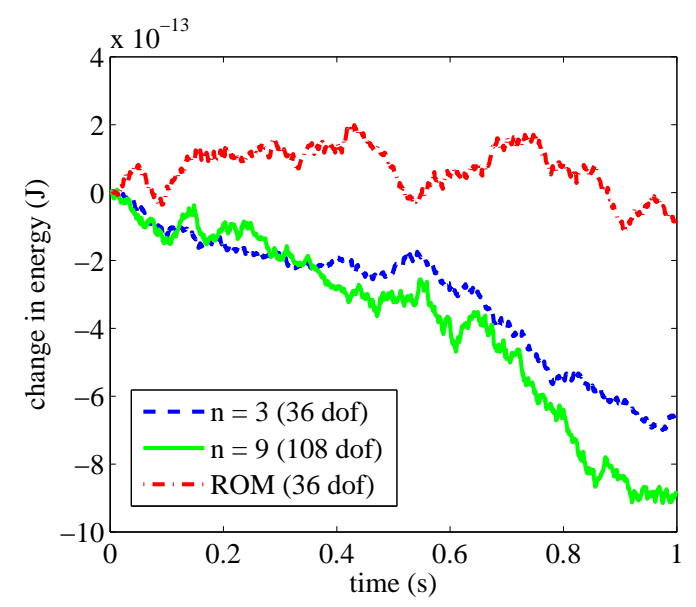

(f)

Figure 4: Nonlinear simulation of beam : (a) Tip vertical velocity; (b) Horizontal bending moment at the root; (c) Root twisting moment; (d) Root vertical velocity; (e) Energy; (f) Change in energy 